\title{
Article \\ A New Development of the Classical Single Ladder Problem via Converting the Ladder to a Staircase
}

\author{
Ralph Høibakk ${ }^{1}$, Dag Lukkassen ${ }^{1}$, Annette Meidell ${ }^{1, *}$ and Lars-Erik Persson ${ }^{1,2}$ \\ 1 Department of Computer Science and Computational Engineering, UiT The Arctic University of Norway, \\ 8514 Narvik, Norway; hoibakk@yahoo.no (R.H.); Dag.Lukkassen@uit.no (D.L.); lpe090@post.uit.no (L.-E.P.) \\ 2 Department of Mathematics and Computer Science, Karlstad University, 65188 Karlstad, Sweden \\ * Correspondence: annette.meidell@uit.no
}

check for updates

Citation: Høibakk, R.; Lukkassen, D.; Meidell, A.; Persson, L.-E. A New Development of the Classical Single Ladder Problem via Converting the Ladder to a Staircase. Mathematics 2021, 9, 339. https://doi.org/ $10.3390 /$ math9040339

Academic Editors: Alberto Cabada and William Guo

Received: 29 April 2020

Accepted: 9 December 2020

Published: 8 February 2021

Publisher's Note: MDPI stays neutral with regard to jurisdictional claims in published maps and institutional affiliations.

\section{Copyright: (c) 2021 by the authors.} Licensee MDPI, Basel, Switzerland. This article is an open access article distributed under the terms and conditions of the Creative Commons Attribution (CC BY) license (https:// creativecommons.org/licenses/by/ $4.0 /)$.

\begin{abstract}
Our purpose is to shed some new light on problems arising from a study of the classical Single Ladder Problem (SLP). The basic idea is to convert the SLP to a corresponding Single Staircase Problem. The main result (Theorem 1) shows that this idea works fine and new results can be obtained by just calculating rational solutions of an algebraic equation. Some examples of such concrete calculations are given and examples of new results are also given. In particular, we derive a number of integer SLPs with congruent ladders, where a set of rectangular boxes with integer sides constitutes a staircase along a common ladder. Finally, the case with a regular staircase along a given ladder is investigated and illustrated with concrete examples.
\end{abstract}

Keywords: single ladder problem (SLP); integer parametric solutions; single staircase problem; quartic equations; algebraic equations; recreational mathematics

MSC: 65H04; 11D09; 00A08

\section{Introduction}

It is well known (see, e.g., [1]) that the solutions to the quartic equations

$$
z^{4}-2 L z^{3}+\left(L^{2}-a^{2}-b^{2}\right) z^{2}+2 L a^{2} z-L^{2} a^{2}=0
$$

give solutions to the Single Ladder Problem (SLP). This problem reads: A ladder of length $L$ is raised to a wall and touches the corner of a rectangular box with sides $a$ and $b$ (see Figure 1 , where also the notations $z$ and $y$ below are defined). The original problem is, for given $a$, $b$, and $L$, to algebraically determine the distance of the ladder between the corner of the rectangle and the wall. In this paper we are looking for integer solutions.

The quartic Equation (1) follows from eliminating the variable $y$ from the two simultaneous second-degree equations that can be formulated from Figure 1:

$$
\begin{aligned}
z^{2} & =a^{2}+(y-b)^{2}, \\
\frac{z}{y-b} & =\frac{L-z}{b} .
\end{aligned}
$$

If $a=b$, then the quartic equations can, by algebraic manipulations, be reduced to solving quadratic equations. If $a \neq b$, then we need to find an algebraic solution to the quartic, which again requires the use of the traditional and cumbersome methods for solving quartics.

Let $L_{\min }$ denote the minimum length of the ladder in a quadrant one for a given set $a>0$ and $b>0$. It has been shown that if $L>L_{\min }$, then the problem has four real solutions - two positions for $L$ in the first quadrant, one in the second quadrant and one in the fourth quadrant; if $L=L_{\mathrm{min}}$, then we have double real solutions in the first quadrant 
and one real solution in each of quadrants two and four, and if $L<L_{\min }$, then there are two complex roots in the first quadrant and one real solution in each of the quadrants two and four, see [2] and cf. also Figures 2 and 3.

\section{Single Ladder Problem}

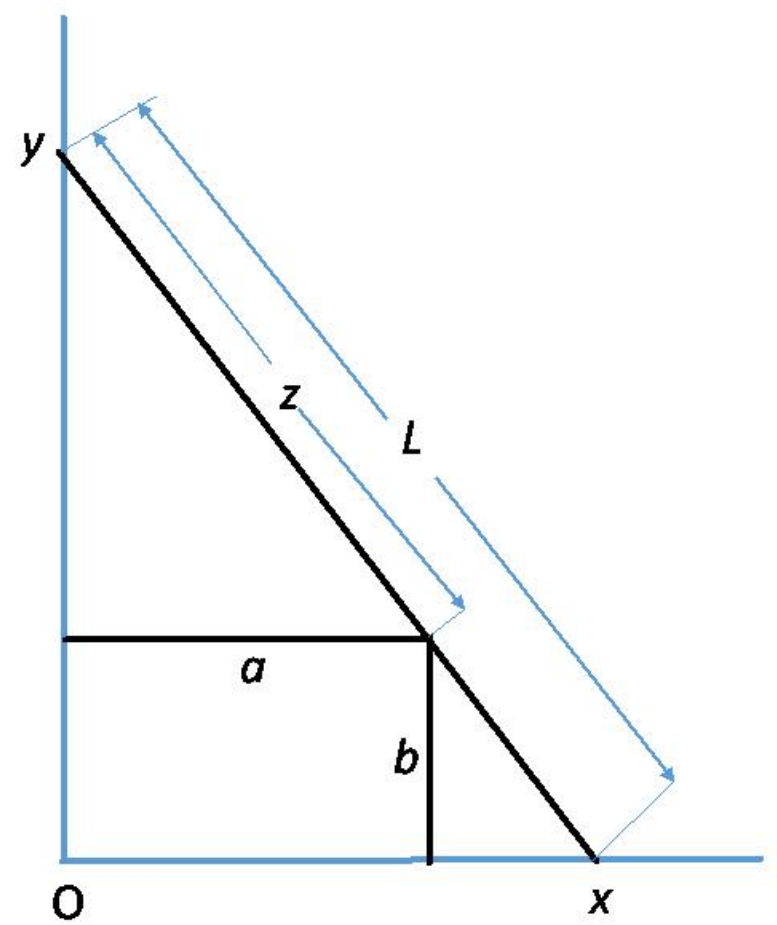

Figure 1. Single Ladder Problem (SLP): Calculate $z$ as a function of $a, b$ and $L$.

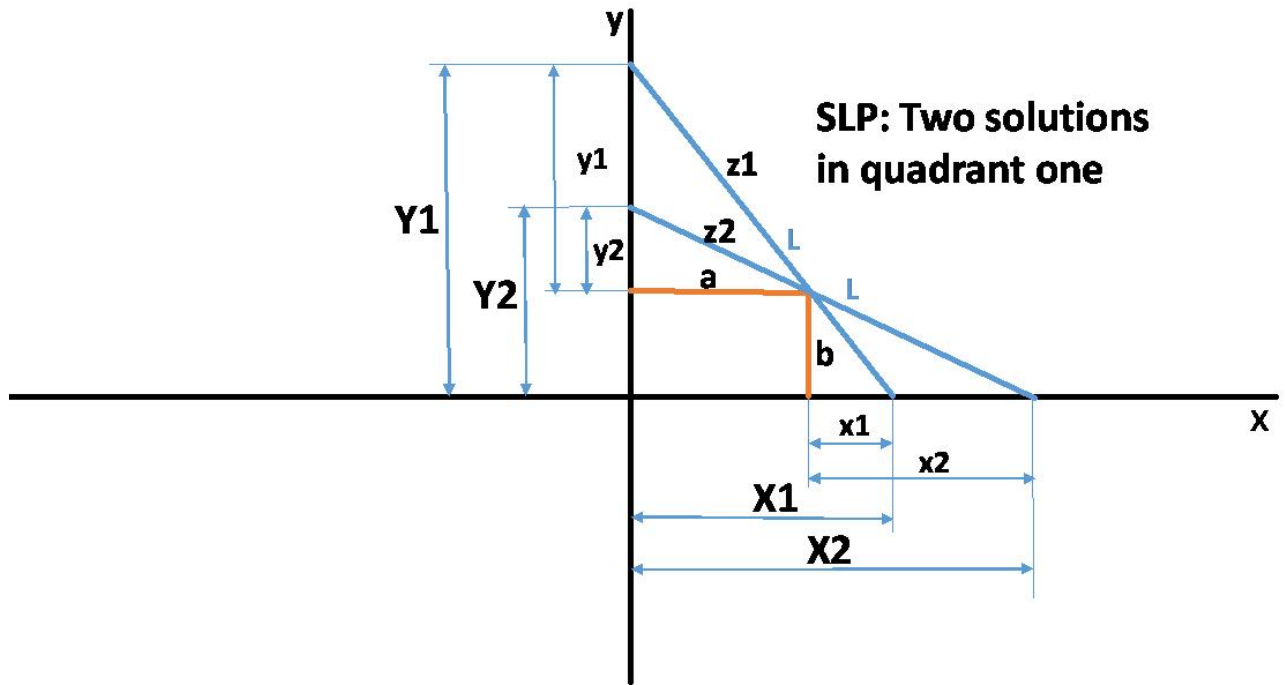

Figure 2. SLP with two solutions in quadrant 1. 


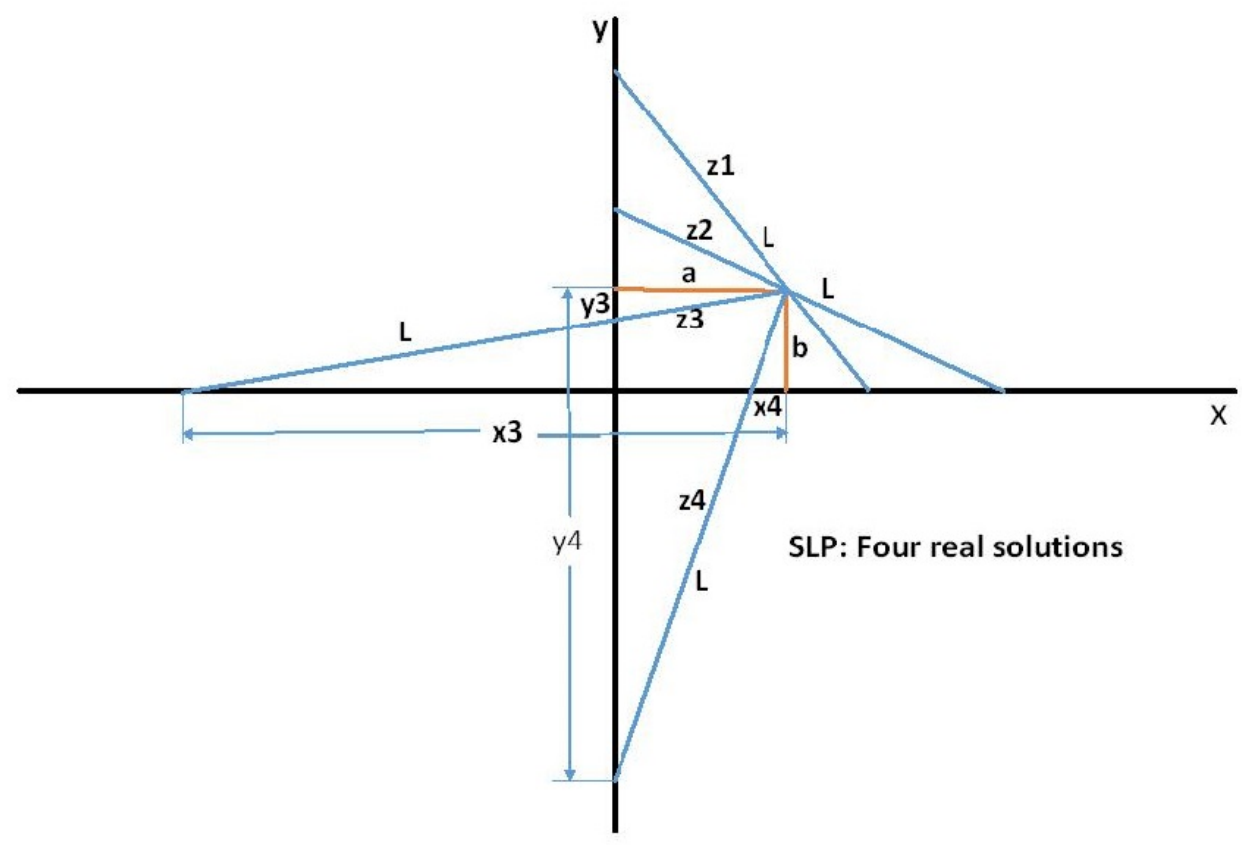

Figure 3. SLP with four solutions, two in quadrant 1 and one in each of quadrants 2 and 4 .

Historically the problem has attracted great interest of professional mathematicians as an algebraic exercise. The first reference to work on similar problems dates back to classic Greek mathematicians, namely to Apollonius; see, e.g., [1]. In the late renaissance or birth of modern mathematics, Descartes and Newton published several works considering variations over the same basic problem, see, e.g., [3,4]. In the eighteenth century Simpson and Lagrange included work on this problem in textbooks on algebra; see $[5,6]$. The solutions presented in these works were algebraic solutions to variations of the quartic equation.

Later on, the Single Ladder Problem was a very popular subject in both professional and recreational mathematics and a number of papers have been published demonstrating real solutions to different versions of the problem; see, e.g., [7-15] and also the recent especially illuminating review article [16] by Coxeter. Several of these developments have concentrated on the attempt to find integer solutions of the SLP. In this paper we concentrate on this interesting case.

In a recently published article [17], the authors redefined the SLP to be regarded as a Diophantine problem and showed a method for finding integer parametric solutions to the quartic equation in question, both for the solutions in quadrant one and for all four solutions. The solution for integer variables in quadrant one is shown in (2).

The research on the SLP has so far been restricted to only one rectangular box with sides $a$ and $b$ and to the two companion geometrical positions for the ladder $L$ of identical lengths in quadrant one.

The purpose of this paper is to continue the Diophantine avenue initiated in [17] and to investigate:

(a) (Section 2): If a number of integer rectangular boxes with sides $a_{i}$ and $b_{i}$ can touch a common integer ladder $L$ with a fixed position in quadrant one, constituting a staircase along the ladder, see Figure 4;

(b) (Section 3): If the variables $a_{i}$ and $b_{i}$ of the rectangular boxes defined in (a) can be adjusted to constitute a ladder with regular intervals along the common ladder, see Figure 5; and

(c) (Section 4): If a number of integer rectangular boxes with variables $a_{i}$ and $b_{i}$ can individually touch integer ladders $L_{i}$ with identical lengths but in different geometrical position in quadrant one; see Figure 6. 
In a sense this means that the Single Ladder Problem is converted into a corresponding staircase problem we have chosen to call the "Single Staircase Problem".

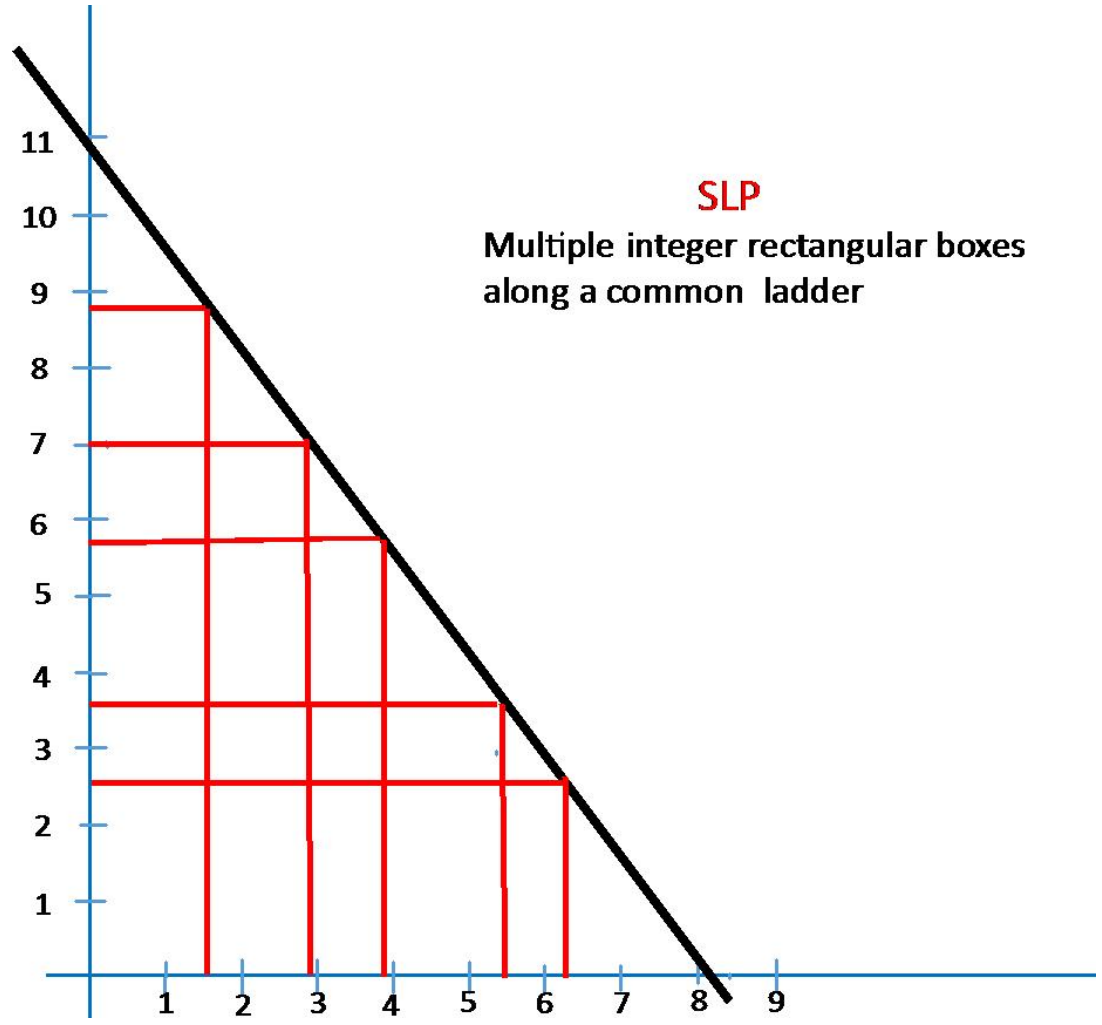

Figure 4. Five SLPs with congruent ladders in quadrant 1.

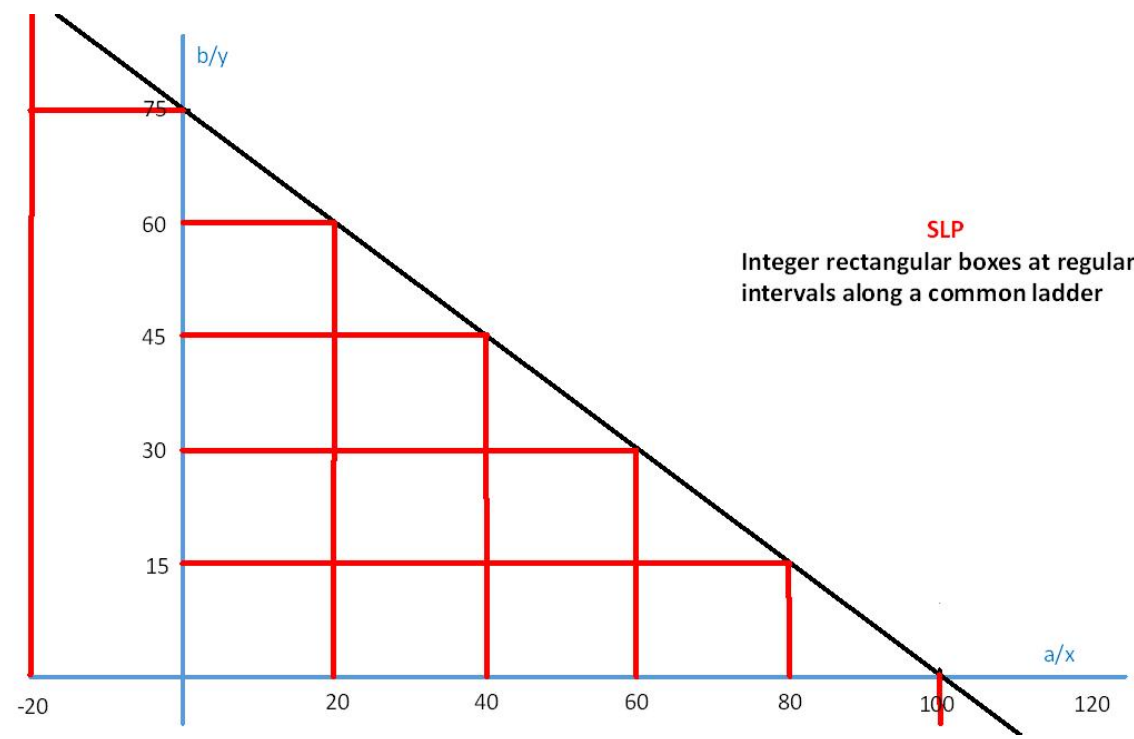

Figure 5. SLP Integer rectangular boxes at regular intervals along a common ladder. 


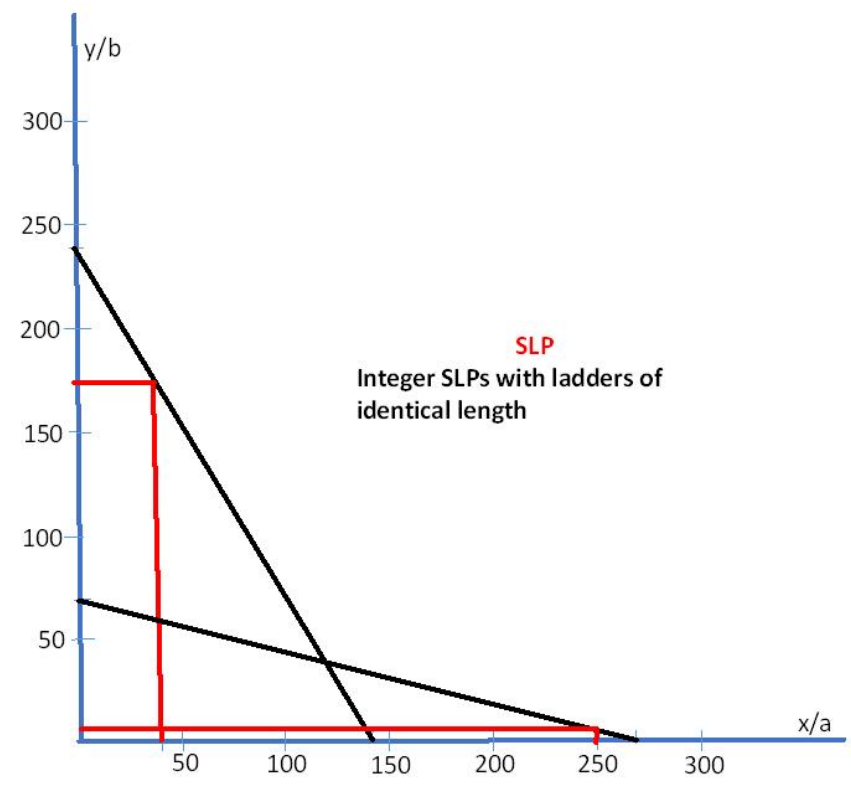

Figure 6. Integer SLPs with ladders of equal lengths.

\section{Integer Solutions of SLPs with Rectangular Boxes Touching a Given SLP Ladder}

The complete parametric integer solutions to the SLP in the first quadrant, which were recently derived in [17], are shown in (2) below.

$$
\begin{aligned}
a & =4 r s t w(s t+r w) \\
b & =(r t-s w)\left(r^{2}-s^{2}\right)\left(t^{2}-w^{2}\right) \\
L & =\left(t^{2}+w^{2}\right)\left(r^{2}+s^{2}\right)(r t+s w), \\
x_{1} & =2 r s(r t-s w)\left(t^{2}-w^{2}\right) \\
x_{2} & =2 t w(r t-s w)\left(r^{2}-s^{2}\right) \\
y_{1} & =2 t w(s t+r w)\left(r^{2}-s^{2}\right) \\
y_{2} & =2 r s(s t+r w)\left(t^{2}-w^{2}\right) \\
z_{1} & =2 t w(s t+r w)\left(r^{2}+s^{2}\right) \\
z_{2} & =2 r s(s t+r w)\left(t^{2}+w^{2}\right) \\
L-z_{1} & =\left(t^{2}-w^{2}\right)\left(r^{2}+s^{2}\right)(r t-s w) \\
L-z_{2} & =\left(t^{2}+w^{2}\right)\left(r^{2}-s^{2}\right)(r t-s w),
\end{aligned}
$$

and define:

$$
\begin{aligned}
& X_{1}:=x_{1}+a=2 r s\left(t^{2}+w^{2}\right)(r t+s w) \\
& X_{2}:=x_{2}+a=2 t w\left(r^{2}+s^{2}\right)(r t+s w) \\
& Y_{1}:=y_{1}+b=\left(t^{2}+w^{2}\right)\left(r^{2}-s^{2}\right)(r t+s w) \\
& Y_{2}:=y_{2}+b=\left(t^{2}-w^{2}\right)\left(r^{2}+s^{2}\right)(r t+s w),
\end{aligned}
$$

where the parameters $r>s$ and $t>w$ are positive integers. The variables $a, b, L, x_{i}, y_{i}, z_{i}$, $X_{i}$ and $Y_{i}, i=1,2$, are illustrated in Figure 2 . 
In the following we use the suffix $i$ in $S L P_{i}$ to denote the $i$-th SLP and the plural $s$ in $S L P S$ to indicate a number of SLPs.

We know that an $S L P_{1}$ that can be determined from (2) will have two integer solutions for $L$ in quadrant one (see Figure 2), i.e., the solution includes two ladders of equal length that will only be identical if:

$$
L_{1}=L_{1 \mathrm{~min}}
$$

for a given set of $a_{1}, b_{1}$. The problem of determining a number of other integer $S L P_{i} s$, where one of the ladders is congruent in space with one of the ladders of $S L P_{1}$ 's, and where the different rectangular boxes constitute a staircase along the common ladder is equivalent to solving a number of simultaneous quartic equations:

$$
\begin{gathered}
z_{1}^{4}-2 L z_{1}^{3}+\left(L^{2}-a_{1}^{2}-b_{1}^{2}\right) z_{1}^{2}+2 L a_{1}^{2} z_{1}-L^{2} a_{1}^{2}=0 \\
z_{2}^{4}-2 L z_{2}^{3}+\left(L^{2}-a_{2}^{2}-b_{2}^{2}\right) z_{2}^{2}+2 L a_{2}^{2} z_{2}-L^{2} a_{2}^{2}=0 \\
z_{3}^{4}-2 L z_{3}^{3}+\left(L^{2}-a_{3}^{2}-b_{3}^{2}\right) z_{3}^{2}+2 L a_{3}^{2} z_{3}-L^{2} a_{3}^{2}=0 \\
\vdots \\
z_{n}^{4}-2 L z_{n}^{3}+\left(L^{2}-a_{n}^{2}-b_{n}^{2}\right) z_{n}^{2}+2 L a_{n}^{2} z_{n}-L^{2} a_{n}^{2}=0
\end{gathered}
$$

In search of solutions to the defined SLP problem, we have formulated a set of requirements that an answer must comply with:

Basic requirement: We assume an $S L P_{1}$ with integer variables $a_{1}, b_{1}, L_{1}$ and will in the following use the position of $L_{1}$ determined by $X_{1}, Y_{1}$ taken from (2). We then seek other integer $S L P_{i} S$ where the points $a_{i}, b_{i}$ lie on the line through $L_{1}$, such that the different rectangular boxes will constitute a staircase along $L_{1}$. The other $S L P_{i} S$ are then required to have one of their $L_{i} s$ equal to $L_{1}$ and to be congruent in space.

Additional requirements: In addition we also initially require that the other integer $S L P_{i} s$, like $S L P_{1}$, have two integer solutions, and that the SLP-variables can be expressed as parametric values of the same set and the same degree in the parametric variables $r, s, t$ and $w$ as $S L P_{1}$. These two requirements have been set in order to see if the system allows a number of integer SLPs that have the same numerical structure and still comply with the basic requirement of a number of rectangular boxes that touch the common ladder.

We will in Section 3 relax the latter two requirements to show how to determine an infinite number of rectangular boxes that touch the common ladder at regular intervals.

In the following we will initially concentrate on searching for integer solutions to the line variables. If the integer solutions are not directly achievable, then we will continue our investigation to determine rational solutions, still requiring that they comply with the additional requirements concerning the numerical structure expressed by the parameters $r, s, t$ and $w$. Rational solutions to the line variables can then ultimately be transformed to integer SLPs by scaling for common factors in the nominators and by multiplying with the least common multiple of the denominators.

From (2) and Figure 2 we see that the line through $L_{1}$ crosses the $x$ - and $y$-axes in $X_{1}=2 r s\left(t^{2}+w^{2}\right)(r t+s w)$ and $Y_{1}=\left(t^{2}+w^{2}\right)\left(r^{2}-s^{2}\right)(r t+s w)$. The line through these points is described by the following formula:

$$
u=-\frac{\left(r^{2}-s^{2}\right)}{2 r s} v+\left(t^{2}+w^{2}\right)\left(r^{2}-s^{2}\right)(r t+s w) .
$$

The point $\left(a_{1}, b_{1}\right)$ from $S L P_{1}$ lies on this line, and we require that the other integers $a_{i}, b_{i}$ from $S L P_{i}$, like $a_{1}, b_{1}$, must be of grade six in the parameters and lie on this line to comply with the basic and additional set of requirements. 


\subsection{The Main Result for This Case}

Theorem 1. All integer solutions to other $S L P_{i} s$, where the point $\left(a_{i}, b_{i}\right)$ lies on the line through $L_{1}$ defined by (1) and where $L_{i}=L_{1}$ are congruent in space, can be found by setting $a_{i}=2 r s F$, where $F$ is any integer function of the parameters, and by finding all integer solutions to the equation:

$$
\begin{gathered}
z^{3}+\left(r^{2}+s^{2}\right)\left(F-2 r t^{3}-2 s w^{3}-2 r t w^{2}-2 s t^{2} w\right) z^{2} \\
-4 r^{2} s^{2}\left(t^{2}+w^{2}\right)(r t+s w)\left(2 F-r t^{3}-s w^{3}-r t w^{2}-s t^{2} w\right) z \\
+4 F r^{2} s^{2}\left(t^{2}+w^{2}\right)^{2}\left(r^{2}+s^{2}\right)(r t+s w)^{2}=0 .
\end{gathered}
$$

Proof. It follows from (4) that the corresponding integer values of $v=a_{i}$ and $u=b_{i}$ can be determined by setting

$$
v=a_{i}=2 r s F,
$$

where $F$ is a function in the parameters. If $F$ is an integer, the corresponding values of $a_{i}$ and $b_{i}$ will also be integers. For $a_{i}$ to be of grade six in the parameters, as required by the above additional requirement, $F$ must be a function of fourth degree in the parameters. From (4) it follows that:

$$
u=b_{i}=-\left(r^{2}-s^{2}\right)\left(F-r t^{3}-s w^{3}-r t w^{2}-s t^{2} w\right)
$$

Since we require that $L_{i}=L_{1}$ and to be congruent in space (see (2)), we must have that:

$$
X_{i}=x_{i}+a_{i}=x_{1}+a_{1}
$$

and:

$$
Y_{i}=y_{i}+b_{i}=y_{1}+b_{1}
$$

By now using the values for $a_{i}$ and $b_{i}$ from (6) and (7) and the parametric values for $X_{i}$ and $Y_{i}$ from (2), we can deduce that:

$$
x_{i}=X_{i}-a_{i}=2 r s\left(t^{2}+w^{2}\right)(r t+s w)-2 r s F=-2 r s\left(F-r t^{3}-s w^{3}-r t w^{2}-s t^{2} w\right),
$$

and:

$y_{i}=Y_{i}-b_{i}=\left(t^{2}+w^{2}\right)\left(r^{2}-s^{2}\right)(r t+s w)-\left(-\left(r^{2}-s^{2}\right)\left(F-r t^{3}-s w^{3}-r t w^{2}-s t^{2} w\right)\right)=F\left(r^{2}-s^{2}\right)$

To determine $z_{i}$ and $L_{i}-z_{i}$ we use the fact that from Figure 1 we easily see that:

$$
z_{i}^{2}=y_{i}^{2}+a_{i}^{2}
$$

and that:

$$
\left(L_{i}-z_{i}\right)^{2}=x_{i}^{2}+b_{i}^{2} .
$$

Using the values from (4) and (9) we obtain that:

$$
z_{i}^{2}=F^{2}\left(r^{2}+s^{2}\right)^{2}
$$

and:

$$
\left(L_{i}-z_{i}\right)^{2}=\left(r^{2}+s^{2}\right)^{2}\left(F-r t^{3}-s w^{3}-r t w^{2}-s t^{2} w\right)^{2}
$$

Hence,

$$
z_{i}=F\left(r^{2}+s^{2}\right)
$$

and:

$$
L_{i}-z_{i}=-\left(r^{2}+s^{2}\right)\left(F-r t^{3}-s w^{3}-r t w^{2}-s t^{2} w\right) .
$$


This leads to that:

$$
L_{i}=z_{i}+\left(L-z_{i}\right)=F\left(r^{2}+s^{2}\right)+\left(-\left(r^{2}+s^{2}\right)\left(F-r t^{3}-s w^{3}-r t w^{2}-s t^{2} w\right)\right)=\left(t^{2}+w^{2}\right)\left(r^{2}+s^{2}\right)(r t+s w)
$$

i.e., we have obtained the required equality:

$$
L_{i}=L_{1}
$$

We have now from (2), (6) and (7) an $S L P_{i}$, where:

$$
\begin{aligned}
& \left(a_{i}, b_{i}, L_{i}\right)=\left(2 r s F,-\left(r^{2}-s^{2}\right)\left(F-r t^{3}-s w^{3}-r t w^{2}-s t^{2} w\right),\left(t^{2}+w^{2}\right)\left(r^{2}+s^{2}\right)(r t+s w)\right) \\
& \text { Moreover, from (8), (9), (12) and (13) it follows that: } \\
& \qquad\left(x_{i}, y_{i}, z_{i},(L-z)_{i}\right)= \\
& \left(-2 r s\left(F-r t^{3}-s w^{3}-r t w^{2}-s t^{2} w\right), F\left(r^{2}-s^{2}\right), F\left(r^{2}+s^{2}\right),-\left(r^{2}+s^{2}\right)\left(F-r t^{3}-s w^{3}-r t w^{2}-s t^{2} w\right)\right)
\end{aligned}
$$

It is clear that $\left(a_{i}, b_{i}\right)$ lies on the line through $L_{1}$, where $L_{i}=L_{1}$, and that they are congruent in space.

By varying $F$ in the $S L P_{i}$ defined by (14) we clearly can calculate an infinite number of integer $S L P_{i} S$ that have an $L_{i}$ that are equal to, and that are congruent in space to, $L_{1}$. However, for an $S L P_{i}$, as required by the additional requirements stated above, to have two integer solution like $S L P_{1}$, the $z_{i}$ value of such a solution must also be a solution to (1). $z_{i}$ is the part of the ladder $L_{i}$ that corresponds to the variable $z$ in (1) (see Figure 1) and must therefore be a solution to the quartic. When we insert $\left(a_{i}, b_{i}, L_{i}\right)$ into (1), we must expect the value $z_{i}=F\left(r^{2}+s^{2}\right)$ calculated above to be one of the solutions. The factorized result of the insertion is:

$$
\begin{aligned}
& \left(z-F\left(r^{2}+s^{2}\right)\right)\left(z^{3}+\left(r^{2}+s^{2}\right)\left(F-2 r t^{3}-2 s w^{3}-2 r t w^{2}-2 s t^{2} w\right) z^{2}-\right. \\
& 4 r^{2} s^{2}\left(t^{2}+w^{2}\right)(r t+s w)\left(2 F-r t^{3}-s w^{3}-r t w^{2}-s t^{2} w\right) z+ \\
& \left.4 F r^{2} s^{2}\left(t^{2}+w^{2}\right)^{2}\left(r^{2}+s^{2}\right)(r t+s w)^{2}\right) \\
& =0
\end{aligned}
$$

Here we recognize the solution already found above, namely $z_{i}=F\left(r^{2}+s^{2}\right)$. Hence, we can conclude that all other $z_{j}$ that belong to an SLP that fulfill the given requirements must be an integer or rational solution to the equation:

$$
\begin{aligned}
& z^{3}+\left(r^{2}+s^{2}\right)\left(F-2 r t^{3}-2 s w^{3}-2 r t w^{2}-2 s t^{2} w\right) z^{2}- \\
& 4 r^{2} s^{2}\left(t^{2}+w^{2}\right)(r t+s w)\left(2 F-r t^{3}-s w^{3}-r t w^{2}-s t^{2} w\right) z+ \\
& 4 F r^{2} s^{2}\left(t^{2}+w^{2}\right)^{2}\left(r^{2}+s^{2}\right)(r t+s w)^{2} \\
& =0
\end{aligned}
$$

and that all of them, and no others, will be part of an SLP that complies with the requirements. The proof is complete.

\subsection{Some Possibilities to Find Rational and Integer Solutions of (5)}

Next, we concentrate on the problem of how to concretely calculate rational and integer solutions of (5). In fact, there are several ways to determine the integer solutions to (5). One of them is to use the fact that the product of the solutions to an algebraic equation equals the constant element of the equation up to sign $(-1)^{p}$, where $p$ is the degree of the equation in this particular case of interest $p=3$ (see, e.g., [18]). This fact can be seen 
by writing the algebraic equation as $\left(z-z_{2}\right)\left(z-z_{2}\right)\left(z-z_{3}\right)=0$, whose constant term is $-z_{2} \times z_{3} \times z_{3}$. In our case this means that:

$$
z_{2} z_{3} z_{4}=-4 F r^{2} s^{2}\left(t^{2}+w^{2}\right)^{2}\left(r^{2}+s^{2}\right)(r t+s w)^{2}
$$

The result of this is that an integer solution $z_{2}$ of degree six in the parameters must divide

$$
4 F r^{2} s^{2}\left(t^{2}+w^{2}\right)^{2}\left(r^{2}+s^{2}\right)(r t+s w)^{2}
$$

to comply with the requirements.

The number of sixth-degree permutations of the factors in $4 F r^{2} s^{2}\left(t^{2}+w^{2}\right)^{2}\left(r^{2}+s^{2}\right)(r t+s w)^{2}$, not including the factor $F$, is 144 . The number of permutations including $F$, remembering that $F$ is initially required to be of grade four in the parameters, is 36 . We have inserted all permutations into (5). None of them lead directly to an integer solution of the equation, but most of them allow calculation of rational values for $F$ of grade four in the parameters. The conclusion of this result is that integer solutions of grade six in the parameters with $F$ being an integer and that comply with the basic and the additional requirements do not exist.

Going further, we will investigate if allowing $F$ and the line variables to be rational can eventually, by transforming the solutions, lead to integer $S L P_{i} s$ of a higher degree than six. By inspecting (5), we see that inserting any chosen value for $z$ of grade six into the parameters in (5), will give a result where we can calculate a rational value for $F$ of grade four in the parameters that lead to a solution of (5). For instance, we find that if we insert

$$
z=n r s\left(t^{2}+w^{2}\right)(r t+s w)
$$

where $n$ is an integer, in (5), we can can calculate the corresponding value for $F$ that will lead to a solution of (5). We then find that:

$$
F=\frac{n\left(t^{2}+w^{2}\right)(r t+s w)(2 r-n s)(n r-2 s)}{\left(n^{2}+4\right) r^{2}+\left(n^{2}+4\right) s^{2}-8 n r s}
$$

By varying $n$ we can determine an infinite number of different $F$ that we can insert in (14) to give parametric solutions to different $S L P_{i} s$. This means that the number of different solutions to (5), and the number rational solutions, that can be transformed to integer $S L P_{i} s$, and that comply with the given requirements, is infinite. We have chosen below to show with an example how some new $S L P_{i}$ s can be calculated.

Example 1. We set $z_{i}=r^{2} s^{2}\left(t^{2}+w^{2}\right)$, which divides the constant element in (5), and insert this value into (5). The result is:

$$
\begin{gathered}
r^{2} s^{2}\left(t^{2}+w^{2}\right)^{2}\left(-2 r^{3} s^{4} t^{3}+4 r^{4} s^{2} t^{4}+r^{4} s^{4} t^{2}-2 r^{5} s^{2} t^{3}+4 r^{2} s^{4} w^{4}-2 r^{2} s^{5} w^{3}-2 r^{4} s^{3} w^{3}+r^{4} s^{4} w^{2}+\right. \\
F r^{2} s^{4}+F r^{4} s^{2}+4 F r^{4} t^{2}+4 F s^{4} w^{2}+4 F r^{2} s^{2} t^{2}+4 F r^{2} s^{2} w^{2}-2 r^{2} s^{5} t^{2} w+8 r^{3} s^{3} t w^{3}+8 r^{3} s^{3} t^{3} w- \\
\left.2 r^{3} s^{4} t w^{2}-2 r^{4} s^{3} t^{2} w-2 r^{5} s^{2} t w^{2}+4 r^{2} s^{4} t^{2} w^{2}+4 r^{4} s^{2} t^{2} w^{2}-8 F r^{3} s^{2} t-8 F r^{2} s^{3} w+8 F r s^{3} t w+8 F r^{3} s t w\right)=0 .
\end{gathered}
$$

From this we can deduce that:

$$
F=\frac{r^{2} s^{2}\left(t^{2}+w^{2}\right)\left(-2 r t-2 s w+r^{2}\right)\left(2 r t+2 s w-s^{2}\right)}{r^{2} s^{4}+r^{4} s^{2}+4 r^{4} t^{2}+4 s^{4} w^{2}+4 r^{2} s^{2} t^{2}+4 r^{2} s^{2} w^{2}-8 r^{3} s^{2} t-8 r^{2} s^{3} w+8 r s^{3} t w+8 r^{3} s t w} .
$$

Inserting this value for F in (14) and (15), we arrive at: 


$$
\begin{aligned}
& a_{2}=\frac{2 r^{3} s^{3}\left(t^{2}+w^{2}\right)\left(-2 r t-2 s w+r^{2}\right)\left(2 r t+2 s w-s^{2}\right)}{r^{2} s^{4}+r^{4} s^{2}+4 r^{4} t^{2}+4 s^{4} w^{2}+4 r^{2} s^{2} t^{2}+4 r^{2} s^{2} w^{2}-8 r^{3} s^{2} t-8 r^{2} s^{3} w+8 r s^{3} t w+8 r^{3} s t w}, \\
& b_{2}=\frac{-\left(r^{2}-s^{2}\right)\left(t^{2}+w^{2}\right)(r s-2 r t-2 s w)\left(r^{3} t+s^{3} w-r^{2} s^{2}+r s^{2} t+r^{2} s w\right)(r s+2 r t+2 s w)}{r^{2} s^{4}+r^{4} s^{2}+4 r^{4} t^{2}+4 s^{4} w^{2}+4 r^{2} s^{2} t^{2}+4 r^{2} s^{2} w^{2}-8 r^{3} s^{2} t-8 r^{2} s^{3} w+8 r s^{3} t w+8 r^{3} s t w}, \\
& L_{2}=L_{1}=\left(t^{2}+w^{2}\right)\left(r^{2}+s^{2}\right)(r t+s w), \\
& x_{2}=-\frac{2 r s\left(t^{2}+w^{2}\right)\left(r^{3} t+s^{3} w-r^{2} s^{2}+r s^{2} t+r^{2} s w\right)(r s-2 r t-2 s w)(r s+2 r t+2 s w)}{r^{2} s^{4}+r^{4} s^{2}+4 r^{4} t^{2}+4 s^{4} w^{2}+4 r^{2} s^{2} t^{2}+4 r^{2} s^{2} w^{2}-8 r^{3} s^{2} t-8 r^{2} s^{3} w+8 r s^{3} t w+8 r^{3} s t w}, \\
& y_{2}=\frac{r^{2} s^{2}\left(r^{2}-s^{2}\right)\left(t^{2}+w^{2}\right)\left(-2 r t-2 s w+r^{2}\right)\left(2 r t+2 s w-s^{2}\right)}{r^{2} s^{4}+r^{4} s^{2}+4 r^{4} t^{2}+4 s^{4} w^{2}+4 r^{2} s^{2} t^{2}+4 r^{2} s^{2} w^{2}-8 r^{3} s^{2} t-8 r^{2} s^{3} w+8 r s^{3} t w+8 r^{3} s t w}, \\
& z_{2}=\frac{r^{2} s^{2}\left(r^{2}+s^{2}\right)\left(t^{2}+w^{2}\right)\left(-2 r t-2 s w+r^{2}\right)\left(2 r t+2 s w-s^{2}\right)}{r^{2} s^{4}+r^{4} s^{2}+4 r^{4} t^{2}+4 s^{4} w^{2}+4 r^{2} s^{2} t^{2}+4 r^{2} s^{2} w^{2}-8 r^{3} s^{2} t-8 r^{2} s^{3} w+8 r s^{3} t w+8 r^{3} s t w}, \\
& L_{2}-z_{2}=-\frac{\left(r^{2}+s^{2}\right)\left(t^{2}+w^{2}\right)\left(r^{3} t+s^{3} w-r^{2} s^{2}+r s^{2} t+r^{2} s w\right)(r s-2 r t-2 s w)(r s+2 r t+2 s w)}{r^{2} s^{4}+r^{4} s^{2}+4 r^{4} t^{2}+4 s^{4} w^{2}+4 r^{2} s^{2} t^{2}+4 r^{2} s^{2} w^{2}-8 r^{3} s^{2} t-8 r^{2} s^{3} w+8 r s^{3} t w+8 r^{3} s t w} \\
& X_{2}=x_{2}+a_{2}=x_{1}+a_{1}=2 r s\left(t^{2}+w^{2}\right)(r t+s w), \\
& \text { and } \\
& Y_{2}=y_{2}+b_{2}=y_{1}+b_{1}=\left(t^{2}+w^{2}\right)\left(r^{2}-s^{2}\right)(r t+s w) .
\end{aligned}
$$

When we have calculated a number of rational $S L P_{i}$ from other chosen $z_{i}$ s and want to arrive at a set of integer $S L P_{i} s$ with congruent $L_{i} s$, we will have to scale them with common factors in the nominators and with the least common multiplier of the denominators.

We still need to verify that the above SLP fulfills the requirement that it has two rational solutions. We do this by inserting the values for $\left(a_{2}, b_{2}, L_{2}\right)$ in (1) and factorizing. We then find two factors of degree one in $z$ that we equal to 0 . We can then calculate two values for $z$ that give solutions to (1); one is the value of $z_{2}$ that we have already found, and the other is:

$$
z_{21}=\frac{r^{2} s^{2}\left(t^{2}+w^{2}\right)\left(r^{2} s^{4}+r^{4} s^{2}+4 r^{4} t^{2}+4 s^{4} w^{2}+4 r^{2} s^{2} t^{2}+4 r^{2} s^{2} w^{2}-8 r^{3} s^{2} t-8 r^{2} s^{3} w+8 r s^{3} t w+8 r^{3} s t w\right)}{r^{2} s^{4}+r^{4} s^{2}+4 r^{4} t^{2}+4 s^{4} w^{2}+4 r^{2} s^{2} t^{2}+4 r^{2} s^{2} w^{2}-8 r^{3} s^{2} t-8 r^{2} s^{3} w+8 r s^{3} t w+8 r^{3} s t w} .
$$

From Figure 2 we see that:

$$
y^{2}=z^{2}-a^{2}
$$

and:

$$
x^{2}=(L-z)^{2}-b^{2} .
$$

Using the values from (16) and (17) we then arrive at the corresponding $x$ and $y$ values: 


$$
\begin{aligned}
& x_{21}=\frac{r^{2} s^{2}\left(t^{2}+w^{2}\right)\left(r^{2}-s^{2}\right)(r s+2 r t+2 s w)^{2}(r s-2 r t-2 s w)^{2}}{r^{2} s^{4}+r^{4} s^{2}+4 r^{4} t^{2}+4 s^{4} w^{2}+4 r^{2} s^{2} t^{2}+4 r^{2} s^{2} w^{2}-8 r^{3} s^{2} t-8 r^{2} s^{3} w+8 r s^{3} t w+8 r^{3} s t w}, \\
& y_{21}=\frac{2 r s\left(t^{2}+w^{2}\right)\left(r^{3} t+s^{3} w-r^{2} s^{2}+r s^{2} t+r^{2} s w\right)\left(2 r t+2 s w-s^{2}\right)\left(-2 r t-2 s w+r^{2}\right)}{r^{2} s^{4}+r^{4} s^{2}+4 r^{4} t^{2}+4 s^{4} w^{2}+4 r^{2} s^{2} t^{2}+4 r^{2} s^{2} w^{2}-8 r^{3} s^{2} t-8 r^{2} s^{3} w+8 r s^{3} t w+8 r^{3} s t w} .
\end{aligned}
$$

The verification is complete.

For the demonstration of a number of integer rectangular boxes that constitute steps in a staircase along the common $L_{i}$, we have chosen five values for $z_{i}$ and calculated the corresponding value for $F_{i}$.

$$
\begin{array}{c|c}
z_{i} & F_{i} \\
\hline F\left(r^{2}+s^{2}\right) & 4 r^{2} s^{2}\left(t^{2}+w^{2}\right) \frac{r t+s w}{\left(r^{2}+s^{2}\right)^{2}} \\
r s\left(t^{2}+w^{2}\right)(r t+s w) & \frac{\left(t^{2}+w^{2}\right)(r-2 s)(2 r-s)(r t+s w)}{-8 r s+5 r^{2}+5 s^{2}} \\
r s\left(t^{2}+w^{2}\right)(r t+s w) & \frac{\left(t^{2}+w^{2}\right)(2 r+s)(r+2 s)(r t+s w)}{8 r s+5 r^{2}+5 s^{2}} \\
4 r s\left(t^{2}+w^{2}\right)(r t+s w) & \frac{4\left(t^{2}+w^{2}\right)(r-2 s)(2 r-s)(r t+s w)}{-8 r s+5 r^{2}+5 s^{2}} \\
2 r s\left(r^{2}+s^{2}\right)(r t+s w) & -\frac{2(r t+s w)\left(r^{2} s-r t^{2}-r w^{2}+s^{3}\right)\left(r s^{2}-s t^{2}-s w^{2}+r^{3}\right)}{2 r^{2} s^{2}+2 t^{2} w^{2}+r^{4}+s^{4}+t^{4}+w^{4}-4 r s t^{2}-4 r s w^{2}}
\end{array}
$$

Based on these values we then determined the parametric rational solutions to the corresponding $S L P_{i}$ and obtained, after transforming to integer valued solutions:

$$
\begin{aligned}
& a_{1}=8 r^{3} s^{3}(r t+s w)\left(t^{2}+w^{2}\right)\left(-8 r s+5 r^{2}+5 s^{2}\right)\left(8 r s+5 r^{2}+5 s^{2}\right) \\
& \left(2 r^{2} s^{2}+2 t^{2} w^{2}+r^{4}+s^{4}+t^{4}+w^{4}-4 r s t^{2}-4 r s w^{2}\right) \\
& b_{1}=(r-s)^{3}(r+s)^{3}(r t+s w)\left(t^{2}+w^{2}\right)\left(-8 r s+5 r^{2}+5 s^{2}\right)\left(8 r s+5 r^{2}+5 s^{2}\right) \\
& \left(2 r^{2} s^{2}+2 t^{2} w^{2}+r^{4}+s^{4}+t^{4}+w^{4}-4 r s t^{2}-4 r s w^{2}\right) \\
& a_{2}=2 r s(r-2 s)(2 r-s)(r t+s w)\left(r^{2}+s^{2}\right)^{2}\left(t^{2}+w^{2}\right)\left(8 r s+5 r^{2}+5 s^{2}\right) \\
& \left(2 r^{2} s^{2}+2 t^{2} w^{2}+r^{4}+s^{4}+t^{4}+w^{4}-4 r s t^{2}-4 r s w^{2}\right) \\
& b_{2}=3\left(r^{2}-s^{2}\right)(r t+s w)\left(r^{2}+s^{2}\right)^{2}\left(t^{2}+w^{2}\right)\left(8 r s+5 r^{2}+5 s^{2}\right) \\
& \left(-r s+r^{2}+s^{2}\right)\left(2 r^{2} s^{2}+2 t^{2} w^{2}+r^{4}+s^{4}+t^{4}+w^{4}-4 r s t^{2}-4 r s w^{2}\right), \\
& a_{3}=2 r s(r+2 s)(2 r+s)(r t+s w)\left(r^{2}+s^{2}\right)^{2}\left(t^{2}+w^{2}\right)\left(-8 r s+5 r^{2}+5 s^{2}\right) \\
& \left(2 r^{2} s^{2}+2 t^{2} w^{2}+r^{4}+s^{4}+t^{4}+w^{4}-4 r s t^{2}-4 r s w^{2}\right),
\end{aligned}
$$




$$
\begin{aligned}
& b_{3}=2 r s(r+2 s)(2 r+s)(r t+s w)\left(r^{2}+s^{2}\right)^{2}\left(t^{2}+w^{2}\right) \\
& \left(-8 r s+5 r^{2}+5 s^{2}\right)\left(2 r^{2} s^{2}+2 t^{2} w^{2}+r^{4}+s^{4}+t^{4}+w^{4}-4 r s t^{2}-4 r s w^{2}\right), \\
& a_{4}=8 r s(r-2 s)(2 r-s)(r t+s w)\left(r^{2}+s^{2}\right)^{2}\left(t^{2}+w^{2}\right) \\
& \left(8 r s+5 r^{2}+5 s^{2}\right)\left(2 r^{2} s^{2}+2 t^{2} w^{2}+r^{4}+s^{4}+t^{4}+w^{4}-4 r s t^{2}-4 r s w^{2}\right), \\
& b_{4}=-3\left(r^{2}-s^{2}\right)(r t+s w)\left(r^{2}+s^{2}\right)^{2}\left(t^{2}+w^{2}\right)\left(8 r s+5 r^{2}+5 s^{2}\right)\left(-4 r s+r^{2}+s^{2}\right) \\
& \left(2 r^{2} s^{2}+2 t^{2} w^{2}+r^{4}+s^{4}+t^{4}+w^{4}-4 r s t^{2}-4 r s w^{2}\right), \\
& a_{5}=-4 r s(r t+s w)\left(r^{2}+s^{2}\right)^{2}\left(-8 r s+5 r^{2}+5 s^{2}\right)\left(8 r s+5 r^{2}+5 s^{2}\right) \\
& \left(r^{2} s-r t^{2}-r w^{2}+s^{3}\right)\left(r s^{2}-s t^{2}-s w^{2}+r^{3}\right), \\
& b_{5}=\left(r^{2}-s^{2}\right)(r t+s w)\left(r^{2}+s^{2}\right)^{2}\left(-8 r s+5 r^{2}+5 s^{2}\right)\left(8 r s+5 r^{2}+5 s^{2}\right)\left(2 r s-t^{2}-w^{2}\right) \\
& \left(r^{2}+s^{2}-t^{2}-w^{2}\right)\left(r^{2}+s^{2}+t^{2}+w^{2}\right), \\
& X_{i}=x_{i}+a_{i}=2 r s(r t+s w)\left(r^{2}+s^{2}\right)^{2}\left(t^{2}+w^{2}\right)\left(-8 r s+5 r^{2}+5 s^{2}\right)\left(8 r s+5 r^{2}+5 s^{2}\right) \\
& \left(2 r^{2} s^{2}+2 t^{2} w^{2}+r^{4}+s^{4}+t^{4}+w^{4}-4 r s t^{2}-4 r s w^{2}\right), \\
& Y_{i}=y_{i}+b_{i}=\left(r^{2}-s^{2}\right)(r t+s w)\left(r^{2}+s^{2}\right)^{2}\left(t^{2}+w^{2}\right)\left(-8 r s+5 r^{2}+5 s^{2}\right)\left(8 r s+5 r^{2}+5 s^{2}\right) \\
& \left(2 r^{2} s^{2}+2 t^{2} w^{2}+r^{4}+s^{4}+t^{4}+w^{4}-4 r s t^{2}-4 r s w^{2}\right), \\
& L_{i}=\left(t^{2}+w^{2}\right)\left(r^{2}+s^{2}\right)(r t+s w)\left(r^{2}+s^{2}\right)^{2}\left(-8 r s+5 r^{2}+5 s^{2}\right)\left(8 r s+5 r^{2}+5 s^{2}\right) \\
& \left(2 r^{2} s^{2}+2 t^{2} w^{2}+r^{4}+s^{4}+t^{4}+w^{4}-4 r s t^{2}-4 r s w^{2}\right) .
\end{aligned}
$$

Each of the SLPs listed in (18) are complete in the sense that they have two integer solutions after transforming the corresponding rational solutions. As we include more solutions, and the number of rectangular boxes, and consequently the number of steps in the stairway increase, the degree as a function of the parameters will increase.

Example 2. In particular, for $r, s, t, w=3,1,3,2$, after scaling for common factors, we get the following integer solutions:

$$
\begin{array}{ll}
a_{1}=2935062 \approx 2.9 \cdot 10^{6} & b_{1}=6957184 \approx 7.0 \cdot 10^{6} \\
a_{2}=1567875 \approx 1.6 \cdot 10^{6} & b_{2}=8780100 \approx 8.8 \cdot 10^{6} \\
a_{3}=3856125 \approx 3.9 \cdot 10^{6} & b_{3}=5729100 \approx 5.7 \cdot 10^{6} \\
a_{4}=6271500 \approx 6.3 \cdot 10^{6} & b_{4}=2508600 \approx 2.5 \cdot 10^{6} \\
a_{5}=5472300 \approx 5.5 \cdot 10^{6} & b_{5}=3574200 \approx 3.6 \cdot 10^{6}
\end{array}
$$




$$
\begin{aligned}
& L_{i}=13588250 \approx 13.6 \cdot 10^{6} \\
& X_{i}=8152950 \approx 8.2 \cdot 10^{6} \\
& Y_{i}=10870600 \approx 10.9 \cdot 10^{6} .
\end{aligned}
$$

The stairway in Example 2 will look approximately as in Figure 6.

Remark 1. All of the parametric points $\left(a_{i}, b_{i}\right)$ lie on the line through $\left(a_{1}, b_{1}\right)$ defined by $(4)$ and the values from (2). The values chosen for the parameters in the numerical example above place the corresponding integer solution points in quadrant one, as shown in Figure 4. Depending on the sign of the calculated $a_{i}$ or $b_{i}$ this might not always be the case, but the resulting $L_{i}$ will always be congruent to $L_{1}$ in quadrant one. We have demonstrated this in Figure 7 , where $a_{2}$ is positive while $b_{2}$ is negative. The second integer solution $L_{i 2}$ resulting from $\left(a_{2}, b_{2}\right)$ will in this case lie in in quadrant three and will have the same length, $L_{2}=L_{1}$. Since the points $\left(a_{i}, b_{i}\right)$ are forced to lie on the line defined by $L_{1}, a_{i}$ and $b_{i}$ cannot both be negative. In fact, the point $\left(a_{1}, b_{1}\right)$ is located in quadrant 1 , and $L$ is a line through $\left(a_{1}, b_{1}\right)$ that crosses the $x$-axis and $y$-axis in quadrant 1 . If $a_{i}$ and $b_{i}$ are both negative, then the point $\left(a_{i}, b_{i}\right)$ will be located in quadrant 3 . Through a point in quadrant 3 it is impossible to draw a line that will cross both the $x$-axis and $y$-axis in quadrant 1.

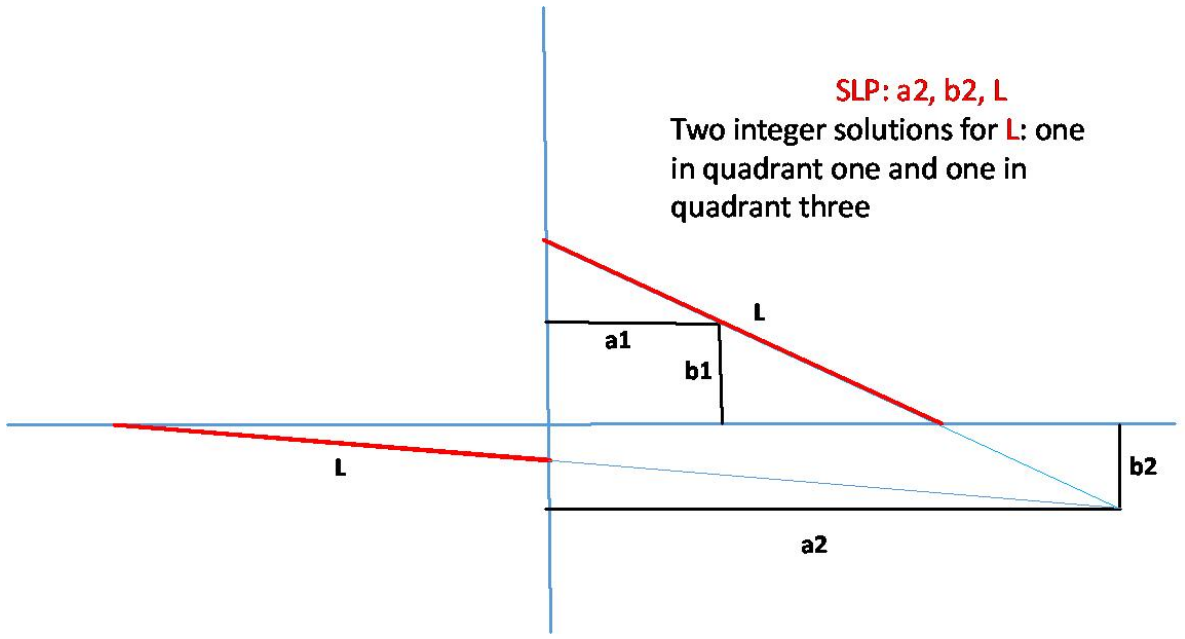

Figure 7. SLP with one ladder in quadrant 1 and one in quadrant 3.

\section{Infinite Regular Staircase along a Given Ladder}

We showed in Section 2 that the number of SLPs that comply with the given requirements is infinite, i.e., we can decide how many rectangular boxes and steps are in the staircase along a given ladder that we want to calculate. However, as can be seen in Figure 4, it is difficult to determine rectangular boxes and the corresponding steps to come at regular intervals.

If we relax the requirements for $S L P_{i}$, for instance by not requiring them to be complete in the sense of two integer solutions, and not demanding that $F$ is of fourth degree in the parameters, then the number of different SLPs, and hence the steps in the stairways, is still infinite, and we are free to arrange the rectangular boxes and the corresponding steps in the staircase to come at regular intervals.

We have from (14) and (15) the following indeterminate $S L P_{i} s$. 


$$
\begin{aligned}
a_{i} & =2 r s F \\
b_{i} & =-\left(r^{2}-s^{2}\right)\left(F-r t^{3}-s w^{3}-r t w^{2}-s t^{2} w\right), \\
x_{i} & =-2 r s\left(F-r t^{3}-s w^{3}-r t w^{2}-s t^{2} w\right), \\
y_{i} & =F\left(r^{2}-s^{2}\right), \\
z_{i} & =F\left(r^{2}+s^{2}\right), \\
L_{i}-z_{i} & =-\left(r^{2}+s^{2}\right)\left(F-r t^{3}-s w^{3}-r t w^{2}-s t^{2} w\right), \\
L_{i} & =L_{1}=\left(t^{2}+w^{2}\right)\left(r^{2}+s^{2}\right)(r t+s w), \\
a_{i}+x_{i} & =a_{1}+x_{1}=2 r s\left(t^{2}+w^{2}\right)(r t+s w),
\end{aligned}
$$

and

$$
b_{i}+y_{i}=b_{1}+y_{1}=\left(t^{2}+w^{2}\right)\left(r^{2}-s^{2}\right)(r t+s w) .
$$

These $S L P_{i} s$ will normally have only one integer solution, but the corner of the box with sides $a_{i}$ and $b_{i}$ will lie on $L_{1}$, and $L_{i}$ will be congruent to $L_{1}$ in space. By choosing $F$ to our liking, we can produce an infinite staircase on the line through $L_{1}$ where the steps come at regular intervals.

$L_{i}$ lies on the line through $X_{i}$ and $Y_{i}$. The angle between this line and the $x$-axis, i.e., between the ladder and the "floor", is:

$$
\alpha=-\operatorname{arctg}\left(\frac{Y_{i}}{X_{i}}\right)
$$

see Figure 2. This will define the steepness $k$ of the staircase along the ladder. If we do not want the staircase to be too steep, say, less than 45 degrees, then we would require that:

$$
|k|=|\operatorname{tg} \alpha|=\left|\frac{Y_{i}}{X_{i}}\right|=\left|\frac{b_{i}+y_{i}}{a_{i}+x_{i}}\right|=\left|\frac{r^{2}-s^{2}}{2 r s}\right|<1 .
$$

By setting $r=2 s$, the steepness factor will be:

$$
|k|=|\operatorname{tg} \alpha|=\frac{3}{4}, \text { and } \alpha=-\operatorname{arctg}\left(\frac{3}{4}\right) \approx|36,9| \text { degrees. }
$$

When we insert this value for $r$ in (19), we find, after scaling for common factors, that the following values define an infinite set of $S L P_{i}$ where the $L_{i} S$ are congruent in space, and where the corresponding staircase along the $L_{i} s$ has a steepness factor of $|k|=\frac{3}{4}$.

$$
\begin{aligned}
& a_{i}=4 F \\
& b_{i}=3 s\left((2 t+w)\left(t^{2}+w^{2}\right)-F\right), \\
& L_{i}=5 s(2 t+w)\left(t^{2}+w^{2}\right), \\
& X_{i}=4 s(2 t+w)\left(t^{2}+w^{2}\right), \\
& Y_{i}=3 s(2 t+w)\left(t^{2}+w^{2}\right) .
\end{aligned}
$$

In particular, by choosing $s, t, w=1,2,1$ we find that:

$$
\left(a_{i}, b_{i}, L_{i}, X_{i}, Y_{i}\right)=(4 F, 3(25-F), 100,75) .
$$


By varying $F$ we can now design a staircase where the depth and height of each step are as we want. For example, by giving $F$ values from -5 to 30 in increments of 5 , we get the staircase shown in Figure 6.

\section{Different SLPs with Ladders of Equal Length}

Another way to look for different SLPs with ladders of equal length is to try and force $L_{1}$ and $L_{2}$ from two different SLPs to become equal. Say we have from (2):

$$
L_{1}=\left(t^{2}+w^{2}\right)\left(r^{2}+s^{2}\right)(r t+s w)
$$

and:

$$
L_{2}=\left(T^{2}+W^{2}\right)\left(R^{2}+S^{2}\right)(R T+S W) .
$$

We then require that:

$$
\left(t^{2}+w^{2}\right)\left(r^{2}+s^{2}\right)(r t+s w)=\left(T^{2}+W^{2}\right)\left(R^{2}+S^{2}\right)(R T+S W) .
$$

We start by simplifying and first ask ourself when:

$$
\left(t^{2}+w^{2}\right)\left(r^{2}+s^{2}\right)=\left(T^{2}+W^{2}\right)\left(R^{2}+S^{2}\right) .
$$

A complete parameter solution for this (see, e.g., [19]) is accomplished by substituting:

$$
\begin{aligned}
t & =(m p+n q), \\
w & =(n p-m q), \\
T & =(m q+n p), \\
W & =(m p-n q), \\
r & =(u z+v x), \\
s & =(v z-u x), \\
R & =(v z+u x), \\
S & =(u z-v x),
\end{aligned}
$$

resulting in:

$$
\left(t^{2}+w^{2}\right)\left(r^{2}+s^{2}\right)=\left(T^{2}+W^{2}\right)\left(R^{2}+S^{2}\right)=\left(p^{2}+q^{2}\right)\left(m^{2}+n^{2}\right)\left(x^{2}+z^{2}\right)\left(u^{2}+v^{2}\right) .
$$

Next we must require from (20) that:

$$
(r t+s w)=(R T+S W) .
$$

When we substitute in this formula the new parameters from (21) we find that:

$((u z+v x)(m p+n q)+(v z-u x)(n p-m q))=((v z+u x)(m q+n p)+(u z-v x)(m p-n q))$, from which we deduce that:

$$
2(p x-q z)(m v-n u)=0 .
$$

Hence, by setting:

$$
x=\frac{q z}{p}
$$

or

$$
m=\frac{n u}{v}
$$


we will have that:

$$
L_{1}=L_{2} .
$$

This leads to a parametric representation of the variables that secures that $L_{1}$ and $L_{2}$ have the same length. In addition, we must also require that $L_{1}$ and $L_{2}$ are congruent in space. To obtain that, $L_{1}$ and $L_{2}$ must both lie on the line through $(a, b)$ and $(A, B)$. This turns out not to be the case.

If we, on the other hand, insert both requirements,

$$
x=\frac{q z}{p} \text { and } m=\frac{n u}{v},
$$

in the formulas for the parameters in (21) and then insert these new parameters into the variables in (2), then we find the following SLPs after scaling for common factors:

$$
\begin{aligned}
a & =8(p u+q v)^{3}(p v-q u)^{3}, \\
b & =(p u+p v-q u+q v)^{3}(p u-p v+q u+q v)^{3}, \\
L_{1} & =\left(p^{2}+q^{2}\right)^{3}\left(u^{2}+v^{2}\right)^{3}, \\
x_{1} & =x_{2}=2(p u-p v+q u+q v)^{2}(p u+p v-q u+q v)^{2}(p u+q v)(p v-q u), \\
y_{1} & =y_{2}=4(p u-p v+q u+q v)(p u+p v-q u+q v)(p u+q v)^{2}(p v-q u)^{2}, \\
x_{1}+a & =2\left(u^{2}+v^{2}\right)^{2}\left(p^{2}+q^{2}\right)^{2}(p u+q v)(p v-q u), \\
y_{1}+b & =\left(u^{2}+v^{2}\right)^{2}\left(p^{2}+q^{2}\right)^{2}(p u+p v-q u+q v)(p u-p v+q u+q v),
\end{aligned}
$$

and

$$
\begin{aligned}
A & =8(p v+q u)^{3}(p u-q v)^{3}, \\
B & =(p u+p v+q u-q v)^{3}(-p u+p v+q u+q v)^{3}, \\
L_{2} & =\left(p^{2}+q^{2}\right)^{3}\left(u^{2}+v^{2}\right)^{3}, \\
X_{1} & =X_{2}=2(p u-p v-q u-q v)^{2}(p u+p v+q u-q v)^{2}(p v+q u)(p u-q v), \\
Y_{1} & =Y_{2}=4(p u+p v+q u-q v)(-p u+p v+q u+q v)(p v+q u)^{2}(p u-q v)^{2}, \\
X_{1}+A & =2\left(u^{2}+v^{2}\right)^{2}\left(p^{2}+q^{2}\right)^{2}(p u-q v)(p v+q u), \\
Y_{1}+B & =\left(u^{2}+v^{2}\right)^{2}\left(p^{2}+q^{2}\right)^{2}(-p u+p v+q u+q v)(p u+p v+q u-q v) .
\end{aligned}
$$

We see from the form of $\left(a, b, L_{1}\right)$ and $\left(A, B, L_{2}\right)$ and the fact that $x_{1}=x_{2}, y_{1}=$ $y_{2}, X_{1}=X_{2}$ and $Y_{1}=Y_{2}$ that both SLPs have $L_{1}$ min and $L_{2}$ min for the given $a, b$ and $A, B$ and that $L_{1}=L_{2}=L$. The same result could have been achieved from (2) by requiring that $L_{1 \mathrm{~min}}=L_{2 \mathrm{~min}}$ in two different SLPs. In fact, one could, by continuing this procedure, produce an infinite number of integer SLPs, where the lengths of the ladders are equal, $L_{1 \min }=L_{2 \min }=\cdots=L_{i \min }=\ldots$, but the different $L_{i} s$ would not be congruent in space.

Example 3. In particular, for the two SLPs above with $(p, q, u, v)=(3,1,3,2)$, we get the following SLPs stairway integer solutions/values (see Figure 6):

$$
\begin{aligned}
\left(a, b, L_{1}, x_{1}+a, y_{1}+b\right) & =(35937,175616,274625,139425,236600) \\
\left(A, B, L_{2}, X_{1}+A, Y_{1}+B\right) & =(250047,4096,274625,266175,67600) .
\end{aligned}
$$




\section{Conclusions}

Let $L_{1}$ be defined by (2) and let $\left(a_{i}, b_{i}\right) \in Z^{+}$be a point on $L_{1}$.The main result of this paper (Theorem 1) gives a precise description regarding how to find all integer solutions of other $\mathrm{SLP}_{i} s$ where $L_{i}=L_{1}$ and they are congruent in space (see Figure 4).

This theorem and the calculations we have performed in this paper show that our reformulation of the classical Singe Ladder Problem via converting the ladder to a staircase is of sense and can be used to obtain genuinely new results.

The crucial point in Theorem 1 is to find integer solutions of the Equation (5). This fact requires new investigations that can imply the corresponding new results. In this paper we contribute by illustrating this fact by giving examples of techniques for how to find integer, and also rational, solutions to Equation (5); see Section 2.2.

Finally, the case with an infinite regular staircase along a given ladder was investigated and also in this case we have illustrated our methods with concrete examples (see Figure 5). As far as we know also the results in these examples are new.

Author Contributions: Writing — review and editing, conceptualization, methodology and writingoriginal manuscript: R.H., D.L., A.M.; project management, supervision and writing-review: R.H., L.-E.P., A.M.; validation: R.H., D.L., A.M., L.-E.P.; data curation, formal analysis: R.H., D.L., L.-E.P.; writing—original draft preparation: R.H., D.L., A.M., L.-E.P.; numerical experiment: L.-E.P., R.H. All authors have read and agreed to the published version of the manuscript.

Funding: This research received no external funding. The APC was funded by UiT The Arctic University of Norway (Faculty of Engineering Science and Technology, Department of Computer Science and Computational Engineering).

Institutional Review Board Statement: Not Appliacable.

Informed Consent Statement: Not Appliacable.

Data Availability Statement: Not Appliacable.

Acknowledgments: We thank all four referees for some generous suggestions, which have improved the final version of this paper.

Conflicts of Interest: The authors declare no conflict of interest.

\section{References}

1. The American Mathematical Monthly; Taylor \& Francis: Abingdon, UK, 1894; pp. 353-354.

2. Holing, K. På gjengrodde stiger, Ladder problems. Normat 1997, 45, 62-78. (In Norwegian)

3. Decartes, R. La Geometrie, (Appendix to His Paper "Discourse on the Method"); Nabu Press: Michigan, MI, USA, 1637.

4. Newton, I. The Mathematical Papers of Isaac Newton; Cambridge University Press: Cambridge, UK, 2008; Volume I, pp. 509-511. 434-439. 202-209. 1667-1670.

5. Lagrange, J.-L. Réflexions sur la resolution algebrique. Nouveaux memoires de l'Academie Royale des Sciences et Belles-Lettres de Berlin 1770, 3, 205-421.

6. Simpson, T. A Treatise of Algebra; John Nourse: London, UK, 1745; p. 250.

7. Cyril Pearson, A. The Twentieth Century Standard Puzzle Book; Geige Routledge \& Sons, Ltd.: New York, NY, USA, 1907 ; p. 103.

8. Holing, K. På gjengrodde stiger-tillegg og rettelser, "Ladder problems-some additions and corrections". Normat 1998, 46, 45. (In Norwegian)

9. Holing, K. På gjengrodde stiger-geometriske løsninger, “Ladder problems-geometric solutions". Normat 2000, 48, 83-90. (In Norwegian)

10. Holing, K. På gjengrodde stiger-epilog, "Ladder problems-epilog”. Normat 2002, 50, 92-95. (In Norwegian)

11. Holing, K. En fjerdegradslikning og dens Galois-gruppe, “A fourth degree equation and its Galios group". Normat 2004, 52, 172-179. (In Norwegian)

12. Sutcliffe, A. Complete Solution of the Ladder Problem in Integers. Math. Gaz. 1963, 47, 133-136. [CrossRef]

13. Baggett, P.; Ehrenfeucht, A. The "Ladder and Box" Problem: From Curves to Calculators, Oral presentation, History and Pedagogy of Mathematics. In Proceedings of the International Mathematical Union (IMU), Deajeon, Korea, 16-20 July 2012.

14. Horwitz, A. A ladder ellipse problem. Forum Geometr. 2016, 16, $73-77$.

15. Wells, D. The "Ladder Problem". In The Penguin Book of Curious and Interesting Puzzles; Penguine Books Ltd.: Middlesex, UK, 1992; p. 130 .

16. Coxeter, H.S.M. The problem of Apollonius. Am. Math. Mon. 2018, 75, 5-15. [CrossRef] 
17. Høibakk, R.; Lukkassen, D.; Meidell, A.; Persson, L.-E. A new look on the Single Ladder Problem (SLP) via integer parametric solutions to the corresponding quartic equation. Mathematics 2020, 8, 267. [CrossRef]

18. Pesic, P. Abel's Proof: An Essay on the Sources of and Meaning of Mathematical Unsolvability; MIT: Cambridge, MA, USA , 2003.

19. Andreescu, T.; Andrica, D.; Cucurezeanu, I. An Introduction to Diophantine Equations: A Problem-Based Approach; Birkhäuser: Basel, Switzerland, 2010; p. 90. 